\title{
The generation of B-mode and circular polarization of cosmic photons due to Non-Commutative space-time background
}

\author{
Seddigheh Tizchang* \\ School of Particles and Accelerators, Institute for Research in Fundamental Sciences (IPM) P.O. \\ Box 19395-5531, Tehran, Iran \\ E-mail: s.tizchangeipm.ir
}

\section{Saghar Batebi}

Department of Physics and Astronomy, University of Western Ontario, London, Ontario,

Canada, N6A $3 K 7$

\section{Mansour Haghighat}

Physics Department, College of Sciences, Shiraz University 71454, Shiraz, Iran

ISC, 71946-94173, Shiraz, Iran

E-mail: M.Haghighateshirazu.ac.ir

\section{Rohoollah Mohammadi}

Iranian National Museum Of Science and Technology (INMOST), P. O. Box 11369-14611,

Tehran, Iran

School of Astronomy, Institute for Research in Fundamental Sciences (IPM), P. O. Box

19395-5531, Tehran, Iran

E-mail: rmohammadieipm.ir

In the standard model of cosmology, the B-mode polarization of the cosmic microwave background can be generated due to the tensor perturbation of metric which is related to gravitational effects in the inflation epoch and scalar perturbation cannot explain B-mode polarization. We consider Compton scattering in the Non-Commutative framework and show that Compton scattering in presence of Non-Commutative background and scalar mode of perturbation, besides generating circularly polarized microwave, can lead to B-mode polarization of the Cosmic Microwave Background. We estimate that generated V-mode $C_{V l}$ can be expressed as linearly polarized power spectrum $C_{P l}$ as well as B-mode power spectrum $C_{B l}$ as a function of $C_{V l}$. We show that $C_{V l}\left(C_{B l}\right)$ for NC-scale $\Lambda_{N C} \sim 20 \mathrm{TeV}$ are in the range of $\mathscr{O}\left(n K^{2}\right)\left(\mathscr{O}\left(p K^{2}\right)\right)$ and higher, respectively, which are in the range of observational data.

The 39th International Conference on High Energy Physics (ICHEP2018)

4-11 July, 2018

Seoul, Korea

${ }^{*}$ Speaker. 
It is believed that the Cosmic Microwave Background (CMB) is partially linearly polarized as a result of the Compton scattering and temperature anisotropies at the time of the last scattering. In the Cosmological Standard Model (CSM), no physical process can generate circular polarization. However, in presence of background fields, circular polarization for the CMB is possible. The Polarization state of the CMB can be explained by well-known Stokes parameters, I, Q, U and V. For a radiation that is linearly polarized, $\mathrm{Q}$ and $\mathrm{U}$ are nonzero. $\mathrm{V}$ indicates circular polarization and for the CMB is usually equal to zero. Moreover, $\mathrm{Q}$ and $\mathrm{U}$ parameters are frame dependent and vary by rotation of the frame. Therefore, it is convenient to define two independent parameters E-mode and B-mode polarizations by using $\mathrm{U}$ and $\mathrm{Q}$ which contain useful information in matter distribution function. density perturbation can only generate E-mode while gravitational wave can produce both. In this paper, we show that Compton scattering in presence of non-trivial background fields such as Non-Commutative (NC) space-time will produce circular and B-mode polarizations by considering scalar metric perturbation. In the NC space-time, it is convenient to characterize the coordinate commentator as:

$$
\left[\hat{x}^{\mu}, \hat{x}^{v}\right]=i \theta^{\mu v} \propto \frac{i}{\Lambda_{N C}^{2}} .
$$

where $\theta^{\mu v}$ is an antisymmetric and real tensor and $\Lambda_{N C}$ represents the NC energy scale. String theory, which motivates the NC space-time, declare that NC scale is of the order of Planck scale: $\Lambda_{N C} \sim M_{P l}$. However, it is shown that by extra dimension formalism, the effect of NC space-time can be reached at TeV scale. Meanwhile, One can build the Non-Commutative Standard Model $(\mathrm{NCSM})$ by the same gauge group as the SM in ordinary space-time: $S U(3)_{c} \times S U(2)_{L} \times U(1)_{Y}$, and the number of particles is just like the SM. To do this the ordinary product are substituted by star product [1] and NC fields are replaced by Seiberg-Witten maps [1]. In this approach, the SM vertices are modified and many new vertices appear.

\section{The CMB polarization in NC space-time}

An ensemble of photons can be characterized by the density matrix $\rho$ which can be explained as a function of four Stokes parameters:

$$
\hat{\rho}=\frac{1}{\operatorname{tr}(\hat{\rho})} \int \frac{d^{3} \mathbf{p}}{(2 \pi)^{3}} \rho_{i j}(\mathbf{p}) D_{i j}(\mathbf{p}), \quad \rho=\frac{1}{2}\left(\begin{array}{cc}
I+Q & U-i V \\
U+i V & I-Q
\end{array}\right),
$$

where $D_{i j}(\mathbf{p})=a_{i}^{\dagger}(\mathbf{p}) a_{j}(\mathbf{p})$ is the photon number operator. The time evolution of $\rho_{i j}(\mathbf{p})$ as a function of Stokes parameters, which is known as the Boltzmann equation for the density matrix, is as follows [2]:

$$
(2 \pi)^{3} \delta^{3}(0)\left(2 p^{0}\right) \frac{d}{d t} \rho_{i j}(\mathbf{p})=i\left\langle\left[H_{I}^{0}(t), D_{i j}^{0}(\mathbf{p})\right]\right\rangle-\frac{1}{2} \int d t\left\langle\left[H_{I}^{0}(t),\left[H_{I}^{0}(0), D_{i j}^{0}(\mathbf{p})\right]\right]\right\rangle,
$$

where $H_{I}^{0}(t)$ is the interacting Hamiltonian at first order. The first term on the right-handed side in above equation is forward scattering. This term is equal to zero for Compton scattering in the SM [2]. The second term is ordinary collision term, called damping. In what follows we propose that Compton scattering in NC space-time can cause new effects on the CMB polarization. For this 
purpose we consider interacting Hamiltonian density for NCQED interaction at leading order:

$$
\mathscr{H}_{N C Q E D}=-i e: \bar{\psi}\left(\frac{1}{2} \theta^{\mu v \rho}\left[\left(\partial_{v} A_{\mu}\right) \vec{\partial}_{\rho}+i e\left(\partial_{\rho} A_{\mu}\right) A_{v}\right]\right) \psi+\mathscr{O}\left(\theta^{2}\right):,
$$

with $\theta^{\mu v \rho}=\theta^{\mu v} \gamma^{\rho}+\theta^{v \rho} \gamma^{\mu}+\theta^{\rho \mu} \gamma^{v}, A_{\mu}$ is the photon field, $\psi$ is electron field, $\vec{\partial}$ denotes the partial derivative acting on the right side and the normal ordering of the operator product is given by colons. The interacting Hamiltonian is obtained by integrating Eq.1.3 over the full space and forming the scattering matrix, expanding fermion and photon fields with creation $a^{\dagger}, b^{\dagger}$ and annihilation $a, b$ operators. Obtained $H_{I}^{0}$ is substituted in Eq.1.2 and after using some standard QED calculations, the time evaluation of the density matrix is as follows (for more details see Ref [3]):

$$
\frac{d}{d t} \rho_{i j}(\mathbf{k})=-\frac{e^{2} Q_{f}^{2}}{4 m_{f} k^{0}} \int d \mathbf{q} n_{f}(\mathbf{x}, \mathbf{q})\left(\delta_{i s} \rho_{s j}-\delta_{j s} \rho_{i s}\right)\left(q \cdot \theta \cdot \varepsilon_{s} q \cdot \varepsilon_{s}+q \cdot \theta \cdot \varepsilon_{s} q \cdot \varepsilon_{s}\right),
$$

where $n_{f}(\mathbf{x}, \mathbf{q})$ indicates the number density of fermions (cosmic electrons and baryons) with following distributions:

$$
n_{f}(\mathbf{x})=\int \frac{d^{3} \mathbf{q}}{(2 \pi)^{3}} n_{f}(\mathbf{x}, \mathbf{q}), \quad m_{f} v_{i}(\mathbf{x}) n_{f}(\mathbf{x})=\int \frac{d^{3} \mathbf{q}}{(2 \pi)^{3}} q_{i} n_{f}(\mathbf{x}, \mathbf{q}) .
$$

Considering the time-like component of NC parameter $\theta^{0 i}$, the time variation of the Stokes parameters in NC space-time is obtained. The primordial scalar perturbations ( $\mathrm{S}$ ) are expanded in Fourier modes which are presented by wave number $\mathbf{K}$. The coordinate system are chosen as $\mathbf{K} \| \hat{z}$, $\left(\hat{e}_{1}, \hat{e}_{2}\right)=\left(\hat{e}_{\theta}, \hat{e}_{\phi}\right)$ as well as electron and baryon bulk velocity direction $\vec{v}_{e}=\vec{v}_{b} \| \mathbf{K}$. Then temperature anisotropy $\Delta_{I}^{(S)}$, circular polarization $\Delta_{V}^{(S)}$, and linear polarization $\Delta_{P}^{ \pm(S)}=Q^{(S)} \pm i U^{(S)}$ of the CMB radiation can be expanded in an appropriate spin-weighted basis given in Ref [3]. Each scattering and interaction in each plane wave can be described as the transport through a plane parallel medium which leads to the Boltzmann equations as follows:

$$
\begin{gathered}
\frac{d}{d \eta} \Delta_{I}^{(S)}+i \mathbf{K} \mu \Delta_{I}^{(S)}+4[\dot{\psi}-i \mathbf{K} \mu \varphi]=C_{e \gamma}^{I}, \quad \frac{d}{d \eta} \Delta_{V}^{(S)}+i \mathbf{K} \mu=C_{e \gamma}^{V}+i \frac{v_{b}}{2}\left[\kappa_{N C}^{-} \Delta_{P}^{+(S)}+\kappa_{N C}^{+} \Delta_{P}^{-(S)}\right] \\
\frac{d}{d \eta} \Delta_{P}^{ \pm(S)}+i \mathbf{K} \mu \Delta_{P}^{ \pm(S)}=C_{e \gamma}^{ \pm}-i v_{b} \kappa_{N C}^{ \pm} \Delta_{V}^{(S)}
\end{gathered}
$$

where $\varepsilon_{\mu i}(k)$ is the four-vector polarization with $i, j, s, s^{\prime}$ running over 1,2 that represent two transverse polarizations of photon. $\mu=\hat{\mathbf{n}} \cdot \hat{\mathbf{K}}=\cos \theta$ is the angle between the wave-vectors and the $\mathrm{CMB}$ photon direction $\hat{\mathbf{n}}=\mathbf{k} /|\mathbf{k}|, C_{e \gamma}$ functions represent the usual Compton scattering contributions to the time evolution of Stokes parameters available at Ref [2], and $\kappa_{N C}$ is defined as follows:

$$
\begin{gathered}
\kappa_{N C}=a(\eta) \frac{3}{4} \frac{\sigma^{T}}{\alpha} \frac{m_{e}^{2}}{\Lambda_{N C}^{2}} \bar{n}_{e} \sum_{f=e, p} \frac{m_{f}}{k^{0}}, \quad \kappa_{N C}^{ \pm}=\kappa_{N C}(C \pm i D), \\
C=-\hat{\theta}^{0 i}\left(\varepsilon_{1 i} v_{f} \cdot \varepsilon_{2}+\varepsilon_{2 i} v_{f} \cdot \varepsilon_{1}\right), \quad D=\hat{\theta}^{0 i}\left(\varepsilon_{1 i} v_{f} \cdot \varepsilon_{1}-\varepsilon_{2 i} v_{f} \cdot \varepsilon_{2}\right),
\end{gathered}
$$

where $a(\eta)$ is the normalized scale factor. By integrating the Boltzmann equations (Eq.1.6) along the line of sight and summing over all Fourier modes, the values of $\Delta_{P}^{ \pm(S)}(\hat{\mathbf{n}})$ and $\Delta_{V}^{(S)}(\hat{\mathbf{n}})$ at the present time $\tau_{0}$ and the direction $\hat{\mathbf{n}}$ can be obtained as

$$
\Delta_{P}^{ \pm(S)}(\hat{\mathbf{n}})=\int d^{3} \mathbf{K} \xi(\mathbf{K}) e^{ \pm 2 i \phi_{K, n}} \Delta_{P}^{ \pm(S)}(\mathbf{K}, \mathbf{k}, \tau), \quad \Delta_{V}^{(S)}(\hat{\mathbf{n}})=\int d^{3} \mathbf{K} \xi(\mathbf{K}) \Delta_{V}^{(S)}(\mathbf{K}, \mathbf{k}, \tau),
$$


with a random variable $\xi(\mathbf{K})$ using to characterize the initial amplitude of the mode and

$$
\begin{aligned}
\Delta_{P}^{ \pm(S)}\left(\mathbf{K}, \mu, \tau_{0}\right) & =\int_{0}^{\tau_{0}} d \tau \dot{\tau}_{e \gamma} e^{i x \mu-\tau_{e \gamma}}\left[\frac{3}{4}\left(1-\mu^{2}\right) \Pi(\mathbf{K}, \tau)-i v_{b} \frac{\kappa_{N C}^{ \pm}}{\dot{\tau}_{e \gamma}} \Delta_{V}^{(S)}\left(\mathbf{K}, \mu, \tau_{0}\right)\right], \\
\Delta_{V}^{(S)}\left(\mathbf{K}, \mu, \tau_{0}\right) & \approx \frac{1}{2} \int_{0}^{\tau_{0}} d \tau \dot{\tau}_{e \gamma} e^{i x \mu-\tau_{e \gamma}}\left[3 \mu \Delta_{V 1}^{(S)}+2 i v_{b} C \frac{\kappa_{N C}}{\dot{\tau}_{e \gamma}} \Delta_{P}^{(S)}\left(\mathbf{K}, \mu, \tau_{0}\right)\right]
\end{aligned}
$$

where the explicit form of $\Delta_{V 1}^{(S)}, \Delta_{P}^{(S)}, \Pi(\mathbf{K}, \tau)$, total and differential optical depth $\tau_{e \gamma}, \dot{\tau}_{e \gamma}$ are given in Ref [3]. The power spectrum $C_{V l}$ in presence of scalar perturbation would be

$$
C_{V l}=\frac{1}{2 l+1} \sum_{m}\left\langle a_{V, l m}^{*} a_{V, l m}\right\rangle \approx \frac{1}{2 l+1} \int d^{3} \mathbf{K} P_{v}(\mathbf{K}) \sum_{m}\left|\int d \Omega Y_{l m}^{*} \int_{0}^{\tau_{0}} d \tau \dot{\tau}_{e \gamma} e^{i x \mu-\tau_{e \gamma}} C \tilde{\kappa} \Delta_{P}^{(S)}\right|^{2},
$$

and $P_{v}(\mathbf{K})$ is the velocity power spectrum. Approximately, the power spectrum for circular polarization can be estimated in terms of the linearly polarized power spectrum $C_{P l}$ as follows

$$
\tilde{\kappa}_{\min }^{2} C_{P l} \leq C_{V l} \leq \tilde{\kappa}_{\max }^{2} C_{P l}, \quad 0.01 n K^{2} \leq C_{V l} \leq 0.01 \mu K^{2},
$$

considering the experimental value for $C_{P l} \sim 0.1 \mu K^{2}$ for $l<250$ [4], $\tilde{\kappa}_{\max / \min }$ are estimated as

$$
\tilde{\kappa}_{\text {max }}=\frac{3}{4} \frac{m_{e}+m_{p}}{T^{0}} \frac{1}{\alpha} \frac{m_{e}^{2}}{\Lambda_{N C}^{2}} \simeq 3 \times 10^{-1}, \quad \tilde{\kappa}_{\text {min }}=\frac{3}{4} \frac{m_{e}+m_{p}}{T^{l s s}} \frac{1}{\alpha} \frac{m_{e}^{2}}{\Lambda_{N C}^{2}} \simeq 3 \times 10^{-4}
$$

in which $\Lambda_{N C} \sim 20 \mathrm{TeV}, k^{0}=T^{0}$ and $k^{0}=T^{l s s}$ are the energies of the CMB photons at the present time and the last scattering epoch, respectively. Compton scattering in NC background and in presence of scalar perturbation, in addition to generation circular polarization, can generate Bmode which is not allowed in ordinary space-time. E-mode and B-mode polarizations can be explained by a divergence-free part $\left(\Delta_{E}^{(S)}\right)$ and curl free part $\left(\Delta_{B}^{(S)}\right)$ which are defined as

$$
\Delta_{E}^{(S)}(\hat{\mathbf{n}}) \equiv-\frac{1}{2}\left[\bar{\partial}^{2} \Delta_{P}^{+(S)}(\hat{\mathbf{n}})+\check{\partial}^{2} \Delta_{P}^{-(S)}(\hat{\mathbf{n}})\right], \quad \Delta_{B}^{(S)}(\hat{\mathbf{n}}) \equiv \frac{i}{2}\left[\bar{\partial}^{2} \Delta_{P}^{+(S)}(\hat{\mathbf{n}})-\check{\partial}^{2} \Delta_{P}^{-(S)}(\hat{\mathbf{n}})\right],
$$

where $ð$ and $\bar{\delta}$ are spin raising and lowering operators, respectively. We obtain that the B-mode power spectrum depends on the circular polarization power spectrum and alternatively to linear polarization power spectrum which is approximated as

$$
\tilde{\kappa}_{\text {min }}^{4} C_{P l} \leq C_{B l}^{S} \leq \tilde{\kappa}_{\text {max }}^{4} C_{P l}, \quad 10^{-4} p K^{2} \leq C_{B l}^{S} \leq 10^{-4} \mu K^{2},
$$

where the estimation is obtained for $\Lambda_{N C} \sim 20 \mathrm{TeV}$ and comparable with the value of $C_{B l}^{o b} \sim 0.01 \mu K^{2}$ for $l<250[4]$.

\section{References}

[1] X. Calmet, B. Jurco, P. Schupp, J. Wess and M. Wohlgenannt, The Standard model on noncommutative space-time, Eur. Phys. J. C 23 (2002) 363 [hep-ph/0111115].

[2] A. Kosowsky, Cosmic microwave background polarization, Annals Phys. 246 (1996) 49 [astro-ph/9501045].

[3] S. Tizchang, S. Batebi, M. Haghighat and R. Mohammadi, Cosmic microwave background polarization in non-commutative space-time Eur. Phys. J. C 76 (2016) no. 9, 478 [hep-ph/1605.09045].

[4] P. A. R. Ade et al. [Planck Collaboration], Planck 2015 results. XX. Constraints on inflation, Astron. Astrophys 594 (2016) A20 [astro-ph.CO/1502.02114]. 